Article

\title{
Thermal Analysis of a Functionally Graded Coating/Substrate System Using the Approximated Transfer Approach
}

\author{
Hui Wang ${ }^{1, *(\mathbb{D})}$ and Qinghua Qin ${ }^{2, *}$ \\ 1 Department of Engineering Mechanics, Henan University of Technology, Zhengzhou 450001, China \\ 2 Research School of Engineering, Australian National University, Canberra ACT 2600, Australia \\ * Correspondence: huiwang@haut.edu.cn (H.W.); qinghua.qin@anu.edu.au (Q.Q.)
}

Received: 10 September 2018; Accepted: 14 January 2019; Published: 16 January 2019

\begin{abstract}
As a heterogeneous material, functionally graded material (FGM) behaves as continuously changed material properties in certain directions from one composition to another, and hence it has received much more attention for biomedical applications and thermal protections to achieve innovative functions that conventional homogeneous material cannot accomplish. However, due to the particularly small thickness ratio of coating to substrate in practice, the conventional mesh discretization of the coating region is inefficient. To simplify the meshing procedure and increase the efficiency of analysis, the approximated transfer algorithm based on the concept of finite difference is developed for transferring boundary conditions applied on the coating surface to the interface of coating and substrate. As a result, only the substrate with transferred convection boundary conditions needs to be solved numerically, i.e., by the fundamental-solution based hybrid finite element method (HFS-FEM) with high accuracy and feasible polygonal element construction, in which only integrals along the element boundary are evaluated because of the application of fundamental solutions of the problem as kernel functions of interior approximated fields. Finally, numerical experiments including the single-layered, multi-layered and functionally graded coatings are carried out to verify the accuracy and applicability of the present method.
\end{abstract}

Keywords: substrate; functionally graded coating; transfer approach; hybrid finite element

\section{Introduction}

Practically, biomaterials are usually not homogeneous and show certain functional gradation formed by biological adaptation [1]. For instance, the porous bone is graded from the external cortical bone, a dense and stiff structure, to the internal cancellous bone which is a porous one [2]. A similar feature can be observed in bamboo [3] and mollusc shell [4]. Such a functionally graded structure optimizes the material's response to external loading. Therefore, it is of great importance that the artificial structures for implant have similar natural functional gradations. As an example of bone implants for knee joint replacement, a functionally graded interlayer is usually designed to improve the acceptance of artificial implants by living tissues. This can be achieved for titanium implants functionalized with a graded coating [5]. Besides, in the case of dental implants, the functionally graded layers were introduced in the titanium and its alloys to increase osteoconductivity and biomechanical bonding between the implant and the surrounding bony tissue [2]. As an application of the $\mathrm{HA}-\mathrm{TiO}_{2}-\mathrm{Ti}$ functionally graded biocompatible coating system designed by Kumar and Wang for implant applications [6], the hydroxyapatite (HA) powder was mixed with the titanium oxide $\left(\mathrm{TiO}_{2}\right)$ power in different weight percentages to form five thin coating layers and then attached to the Ti6A14V metal substrate to obtain the resulting functionally graded composite. Besides, the multi-layered 
or functionally graded coating film was also used as a thermal barrier coating for protecting the covered substrate under severe thermal-mechanical environments [7-10]. For such multi-layered or functionally graded coating/substrate systems, the prediction of temperature distribution in the covered substrate domain is critical for further analysis of thermal stresses and deformations to control them within acceptable levels.

To analyze the multi-layered coating system or the functionally graded coating (FGC) system, numerical modelling is widely employed, including finite element modelling [11,12] and boundary element modelling $[13,14]$. However, it is observed that the thickness of coating is usually very thin, i.e., within $\mu \mathrm{m}$ scale, compared to the size of the covered substrate domain in millimeter scale. The primary obstacle to the development of efficient and accurate computational modelling for such a thin coating system is that the meshing operation in the thin coating layers becomes very difficult and accuracy and efficiency becomes worse as the thickness of the coating layer decreases dramatically. To deal with such an obstacle, the transfer approximation based on finite difference technique [13] and the nonlinear transformation technique $[14,15]$ were respectively developed for transferring the specified boundary conditions on the coating surface to the interface of the coating layer and substrate so that only the substrate region needs to be solved, instead of the whole coating/substrate system. However, there are a very few studies that focused on thermal analyses of heterogeneous coating systems, i.e., FGC $[6,16,17]$.

This paper pays attention to the first attempt of the thermal analysis of a functionally graded coating/substrate system by combining the approximated transfer algorithm and the hybrid finite element with fundamental solution kernels, an effective numerical tool called fundamental-solution based hybrid finite element method (HFS-FEM), which can be viewed as the combination of finite element method (FEM) [12,18] and boundary element method (BEM) [19-21] and has such inherent advantages as the stiffness equation containing element boundary integrals only, specially-purposed elements, arbitrary polygonal-shaped element construction, and better accuracy over the conventional FEM. However, it is worth noting that the dependence of fundamental solutions of HFS-FEM may limit its application to those problems without the explicit expression of fundamental solutions. Detailed summaries of the development of HFS-FEM and the state-of-the-art can be found in [22,23]. In this study, the approximated transfer algorithm based on finite difference is developed to establish a recurrence formula to treat the multi-layered coating and the functionally graded coating. The applied thermal boundary conditions on the top surface of coating can be transferred to the surface of substrate in convection form and then only the substrate domain is solved by the HFS-FEM to analyze the thermal behavior in it. Finally, the accuracy and applicability of the present method is discussed by several numerical examples involving the single-layered coating, the multi-layered coating and the functionally graded coating.

\section{Problem Statement}

To address the generalization of the present method, a general two-dimensional functionally graded coating/substrate system depicted in Figure 1 is considered in the study, where the thickness of heterogeneous coating and substrate is $h_{\mathrm{c}}$ and $h_{\mathrm{s}}$, respectively. It is assumed that the substrate region is homogeneous and isotropic, while the coating layer is graded along the thickness of it. Besides, it is worth pointing out that the substrate is not limited to the finite domain shown in Figure 1, and the half-infinite substrate domain is also workable with the numerical procedure developed below. 


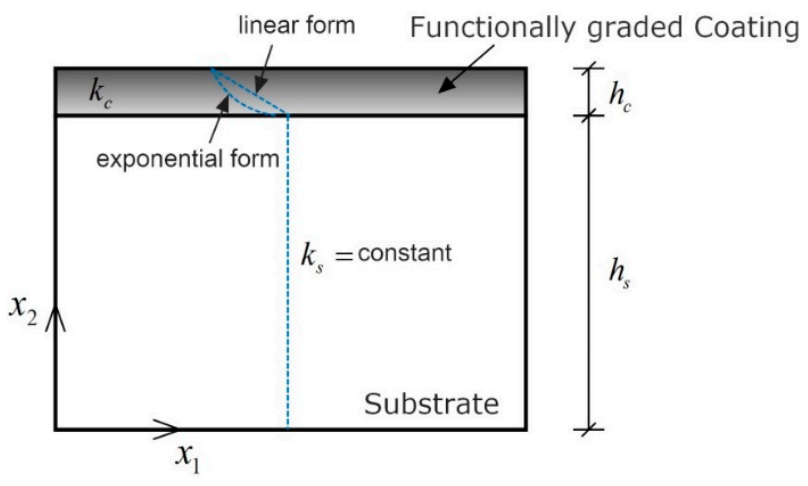

Figure 1. Configuration of the functionally graded coating/substrate system.

For the composite system consisting of the single-layered functionally graded coating and the substrate, the basic partial differential equations at point $x=\left(x_{1}, x_{2}\right)$ governing the steady-state temperature distribution in the coating region $\Omega_{\mathrm{c}}$ and the substrate region $\Omega_{\mathrm{s}}$ can be expressed as:

$$
\begin{array}{ll}
\nabla q_{\mathrm{c}}(x)=0, & x \in \Omega_{\mathrm{c}} \\
\nabla q_{\mathrm{s}}(x)=0, & \mathrm{x} \in \Omega_{\mathrm{s}}
\end{array}
$$

where $q_{\mathrm{c}}$ and $q_{\mathrm{s}}$ are heat flux vectors in the coating and substrate regions, respectively, and can be expressed in terms of temperature variable by the Fourier's law,

$$
\begin{array}{ll}
q_{\mathrm{c}}(x)=-k_{\mathrm{c}} \nabla T_{\mathrm{c}}(x), & x \in \Omega_{\mathrm{c}} \\
q_{\mathrm{s}}(x)=-k_{\mathrm{s}} \nabla T_{\mathrm{s}}(x), & x \in \Omega_{\mathrm{s}}
\end{array}
$$

In Equation (2), $k_{\mathrm{c}}$ and $k_{\mathrm{s}}$ are the thermal conductivity of the coating material and the substrate, respectively. $T_{\mathrm{C}}$ and $T_{\mathrm{S}}$ are the corresponding temperature fields in each region, respectively. For the homogeneous coating, the thermal conductivity $k_{\mathrm{c}}$ is constant, while it may change in terms of spatial coordinates along the thickness of the functionally graded coating. In this study, the following two graded forms are considered, as shown in Figure 1,

$$
k_{\mathrm{c}}\left(x_{2}\right)= \begin{cases}a+b x_{2} & \text { for the linear form } \\ a e^{b x_{2}} & \text { for the exponental form }\end{cases}
$$

where $a$ and $b$ are constant coefficients, which can be determined by the continuous change of thermal conductivity from one material phase to another material phase.

Substituting Equation (2) into Equation (1) yields,

$$
\begin{array}{ll}
\nabla k_{\mathrm{c}} \nabla T_{\mathrm{c}}(x)+k_{\mathrm{c}} \nabla^{2} T_{\mathrm{c}}(x)=0, & x \in \Omega_{\mathrm{c}} \\
k_{\mathrm{s}} \nabla^{2} T_{\mathrm{s}}(x)=0, & x \in \Omega_{\mathrm{S}}
\end{array}
$$

Obviously, the two governing equations defined in Equation (1) are independent of each other, owing to the material difference in thermal conductivity. To couple them, the temperature and normal heat flux continuous conditions at the interface $\Gamma_{i}$ of the coating and substrate should be added for representing local thermal equilibrium:

$$
\begin{array}{lr}
T_{\mathrm{s}}=T_{\mathrm{c}} & \text { on the interface } \Gamma_{i} \\
q_{\mathrm{s}} \cdot n_{\mathrm{s}}+q_{\mathrm{c}} \cdot n_{\mathrm{c}}=0 & \text { on the interface } \Gamma_{i}
\end{array}
$$

where $n_{\mathrm{c}}=-n_{\mathrm{S}}$ are respectively the unit outward normal vector to the interface for the coating domain and substrate domain. 
Typically, the thin functionally graded material (FGM) coating is practically laminated by several homogeneous sublayers with different proportions along its thickness $[6,16]$. For such cases, the governing Equations (4) can be rewritten in general form:

$$
k_{\mathrm{cm}} \nabla^{2} T_{\mathrm{cm}}(x)=0, \quad x \in \Omega_{\mathrm{cm}}, \quad m=1,2, \cdots, M+1
$$

where $k_{\mathrm{cm}}$ is thermal conductivity of the $m^{\text {th }}$ layer, $T_{\mathrm{cm}}$ denotes the temperature in the $m^{\text {th }}$ layer and $M$ is the number of homogeneous sublayers in the functionally graded coating domain, as shown in Figure 2. Note that in Equation (6), the $(M+1)^{\text {th }}$ layer refers to the substrate domain and $k_{\mathrm{c}(M+1)}=k_{\mathrm{s}}$, $T_{\mathrm{c}(M+1)}=T_{\mathrm{S}}, \Omega_{\mathrm{c}(M+1)}=\Omega_{\mathrm{s}}$.

Subsequently, the continuous conditions at the interface of adjacent layers $m$ and $m+1$ can be written as:

$$
\begin{aligned}
& T_{\mathrm{c} m}=T_{\mathrm{c}(m+1)} \\
& q_{\mathrm{c} m} n_{\mathrm{c} m}+q_{\mathrm{c}(m+1)} n_{\mathrm{c}(m+1)}=0
\end{aligned}
$$

where $q_{\mathrm{c} m}=-k_{\mathrm{c} m} \Delta T_{\mathrm{c} m}$ is the heat flux vector in the $m^{\text {th }}(m=1,2, \cdots, M+1)$ layer, and $n_{\mathrm{c} m}=-n_{\mathrm{c}(m+1)}$ is the normal vector to the interface.

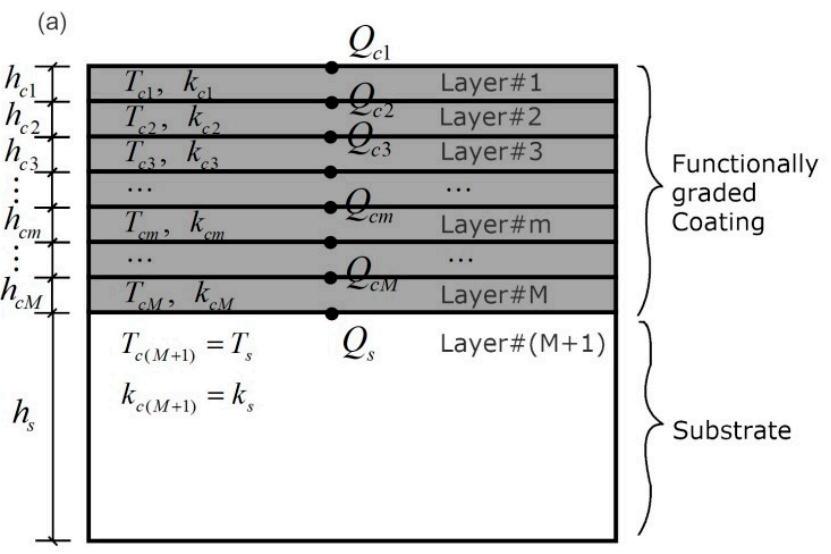

(b)

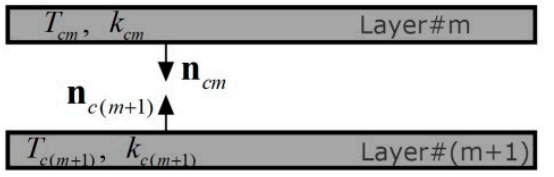

Figure 2. Schematic diagrams of (a) the multi-layered coating/substrate system, and (b) the adjacent layers.

\section{Approximated Transfer Algorithm for Thin Graded Coating Layer}

Conventional multi-region numerical methods such as the FEM and the HFS-FEM are inefficient when the coating is particularly thin. Under such ultra-thin circumstances, extremely refined mesh with massive integration points is required. To tackle this problem, the approximated transfer approach for the single homogeneous coating [13] is firstly reviewed and then is extended to deal with the multi-layered coating and the functionally graded coating by an iterative scheme. Although the temperature distribution in the thin coating domain cannot be given through the present approximated transfer algorithm, the algorithm can significantly improve the computational efficiency in the substrate region, which is particularly interesting to assess the "bioactive" and "protective" effect of the coating.

\subsection{Thin Single-Layered Homogeneous Coating}

For the single-layer homogeneous coating shown in Figure 3, it is assumed that a mixed boundary condition is applied at point $Q_{\mathrm{c}}$,

$$
\left.\alpha T_{\mathrm{c}}\right|_{Q_{\mathrm{c}}}+\left.\beta_{0}\left(q_{\mathrm{c}} \cdot n_{\mathrm{s}}\right)\right|_{Q_{\mathrm{c}}}=\gamma
$$

where $\alpha$ and $\beta_{0}$ are constant coefficients, and $\gamma$ is the given value, respectively. Practically, the combinations $\alpha=1$ and $\beta_{0}=0, \alpha=0$ and $\beta_{0}=1$, and $\alpha \neq 0$ and $\beta_{0} \neq 0$ respectively correspond to the temperature condition, heat flux condition, and convection condition. 
According to the forward finite difference technique [24], the first-derivative of the temperature field $T_{\mathrm{C}}$ at point $Q_{\mathrm{s}}$ can be approximated as:

$$
\left.\frac{\partial T_{\mathrm{c}}}{\partial x_{2}}\right|_{Q_{\mathrm{s}}} \approx \frac{\left.T_{\mathrm{c}}\right|_{Q_{\mathrm{c}}}-\left.T_{\mathrm{c}}\right|_{Q_{\mathrm{s}}}}{h_{\mathrm{c}}}
$$

from which the normal heat flux at point $Q_{\mathrm{s}}$ can be given by:

$$
\left.\left(q_{\mathrm{c}} \cdot n_{\mathrm{c}}\right)\right|_{Q_{\mathrm{s}}}=k_{\mathrm{c}} \frac{\left.T_{\mathrm{c}}\right|_{Q_{\mathrm{c}}}-\left.T_{\mathrm{c}}\right|_{Q_{\mathrm{s}}}}{h_{\mathrm{c}}}
$$

Introducing the interfacial conditions (5) we have:

$$
\left.\left(q_{\mathrm{c}} \cdot n_{\mathrm{c}}\right)\right|_{Q_{\mathrm{s}}}=-\left.\left(q_{\mathrm{s}} \cdot n_{\mathrm{s}}\right)\right|_{Q_{\mathrm{s}}}=k_{\mathrm{c}} \frac{\left.T_{\mathrm{c}}\right|_{Q_{\mathrm{c}}}-\left.T_{\mathrm{s}}\right|_{Q_{\mathrm{s}}}}{h_{\mathrm{c}}}
$$

from which such relation can be derived as:

$$
\left.T_{\mathrm{c}}\right|_{Q_{\mathrm{c}}}=\left.T_{\mathrm{s}}\right|_{Q_{\mathrm{s}}}-\left.\frac{h_{\mathrm{c}}}{k_{\mathrm{c}}}\left(q_{\mathrm{s}} \cdot n_{\mathrm{s}}\right)\right|_{Q_{\mathrm{s}}}
$$

Besides, the heat flux term in Equation (8) is assumed to be same as that applied at the coating substrate interface [13], that is,

$$
\left.\left(q_{\mathrm{c}} \cdot n_{\mathrm{s}}\right)\right|_{\mathrm{Q}_{\mathrm{c}}}=\left.\left(q_{\mathrm{c}} \cdot n_{\mathrm{s}}\right)\right|_{\mathrm{Q}_{\mathrm{s}}}=\left.\left(q_{\mathrm{s}} \cdot n_{\mathrm{s}}\right)\right|_{\mathrm{Q}_{\mathrm{s}}}
$$

Then, substituting Equations (12) and (13) into the mixed boundary condition (8) produces:

$$
\left.\alpha T_{\mathrm{s}}\right|_{Q_{\mathrm{s}}}+\left.\left(\beta_{0}-\frac{\alpha h_{\mathrm{c}}}{k_{\mathrm{c}}}\right)\left(q_{\mathrm{s}} \cdot n_{\mathrm{s}}\right)\right|_{Q_{\mathrm{s}}}=\gamma
$$

from which it is found that the applied boundary condition (8) on the top surface of the coating is approximately transferred to the interface of substrate and coating. Obviously, the results of the specified temperature condition which corresponds to $\alpha=1, \beta_{0}=0$ and the specified heat flux condition which corresponds to $\alpha=0, \beta_{0}=1$ can be regarded as special cases of the general form (14).

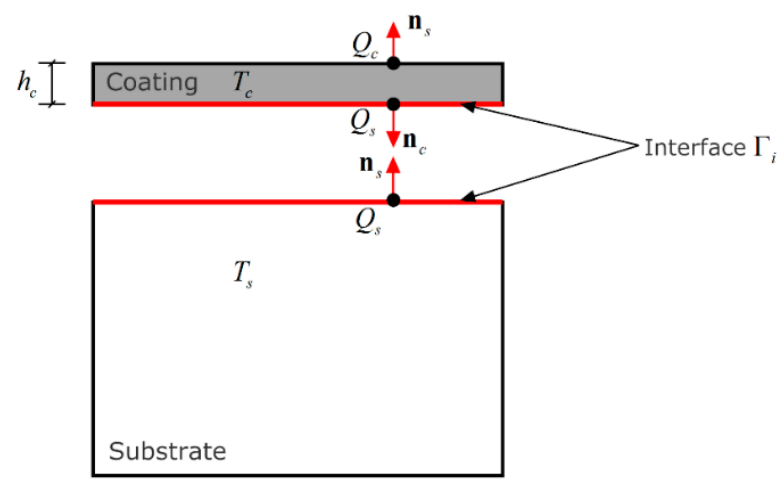

Figure 3. Configuration of the single-layered homogeneous coating/substrate system.

\subsection{Thin Functionally Graded Coating}

Once the approximated transfer approach for the single-layered homogeneous coating is derived, the procedure can be extended for deriving the recurrence formula for the functionally graded coating. In this study, the functionally graded coating is generally divided into $M$ thin layers, and each layer is approximately homogeneous, as shown in Figure 2. It is assumed that the thickness and thermal conductivity of the $m^{\text {th }}$ layer is $h_{\mathrm{c} m}$ and $k_{\mathrm{c} m}(m=1,2, \cdots, M)$, respectively. 
If a general form of boundary condition is applied at the top surface point $Q_{\mathrm{c} 1}$ of the layer \#1,

$$
\left.\alpha T_{\mathrm{c} 1}\right|_{Q_{\mathrm{c} 1}}+\left.\beta_{0}\left(q_{\mathrm{c} 1} \cdot n_{\mathrm{c} 1}\right)\right|_{Q_{\mathrm{c} 1}}=\gamma
$$

by means of the derivation of the single-layered case, the transferred boundary condition at point $Q_{\mathrm{c} 2}$ on the interface of layer \#1 and layer \#2 can be written as:

$$
\left.\alpha T_{\mathrm{s}}\right|_{Q_{\mathrm{c} 2}}+\left.\beta_{1}\left(q_{\mathrm{c} 2} \cdot n_{\mathrm{c} 2}\right)\right|_{Q_{\mathrm{c} 2}}=\gamma
$$

with

$$
\beta_{1}=\beta_{0}-\frac{\alpha h_{\mathrm{c} 1}}{k_{\mathrm{c} 1}}
$$

Following this transferring rule, the resulting thermal-response at the point $Q_{\mathrm{s}}$ on the upper surface of the substrate domain can be written as:

$$
\left.\alpha T_{\mathrm{s}}\right|_{Q_{\mathrm{s}}}+\left.\beta_{M}\left(q_{\mathrm{s}} \cdot n_{\mathrm{s}}\right)\right|_{Q_{\mathrm{s}}}=\gamma
$$

in which the transfer coefficient $\beta_{M}$ is given by the following recurrence relation:

$$
\beta_{m}=\beta_{m-1}-\frac{\alpha h_{\mathrm{c} m}}{k_{\mathrm{c} m}} \quad(m=1, \cdots, M)
$$

Specially, when the temperature condition is applied on the coating surface, that is, $\alpha=1$, $\beta_{0}=0$, Equation (18) reduces to $\left.T_{\mathrm{s}}\right|_{Q_{\mathrm{s}}}+\left.\beta_{M}\left(q_{\mathrm{s}} \cdot n_{\mathrm{s}}\right)\right|_{Q_{\mathrm{s}}}=\gamma$ with $\beta_{M}=-\sum_{m=1}^{M} h_{\mathrm{c} m} / k_{\mathrm{c} m}$. If the normal heat flux condition is given on the coating surface, that is, $\alpha=0, \beta_{0}=1$, Equation (18) reduces to $\left.\left(q_{\mathrm{s}} n_{\mathrm{s}}\right)\right|_{\mathrm{Q}_{\mathrm{s}}}=\gamma$. Besides, the procedure above shows that the present approximated transfer approach is also suitable for multi-layered homogeneous or inhomogeneous coating.

\section{Hybrid Element Formulation}

From the above transfer procedure, it is found that the substrate region should be solved under the approximated convection-typed boundary condition (18) applied on the coating-substrate interface. This can be done through the HFS-FEM including the convection term, which is implemented by introducing fundamental solution kernels for element interior fields and shape functions for element boundary frame field. Compared to the conventional FEM and BEM, and the Trefftz FEM [25,26], this method has some inherent characteristics like stiffness equations containing element boundary integrals only, specially-purposed elements and polygonal-shaped elements and has been successfully applied to elastic $[22,27,28]$ and thermal conduction $[23,29-31]$ problems with better accuracy over the conventional FEM and more flexible multi-sided element construction, prior to this study. Additionally, different to the BEM, the HFS-FEM can be applied to multi-domain or multi-material problems more conveniently.

Before deriving the computing formulation, the mixed boundary condition given in Equation (18) should be reorganized to meet the requirement for implementing the HFS-FEM. In the hybrid variational functional of HFS-FEM for thermal analysis, three types of boundary conditions are involved: specified temperature condition, specified heat flux condition and specified convection condition. For such cases, Equation (18) is rewritten as the following convection form:

$$
\left.\left(q_{\mathrm{s}} \cdot n_{\mathrm{s}}\right)\right|_{Q_{\mathrm{s}}}=\widetilde{h}_{\infty}\left(\left.T_{\mathrm{s}}\right|_{Q_{\mathrm{s}}}-\widetilde{T}_{\infty}\right)
$$

where

$$
\widetilde{h}_{\infty}=-\frac{\alpha}{\beta_{M}}, \quad \widetilde{T}_{\infty}=\frac{\gamma}{\alpha} \quad(\alpha \neq 0)
$$

respectively denote the generalized convection coefficient and environment temperature. 
For two-dimensional steady-state heat transfer in the isotropic homogeneous substrate media, the weak form of the double-variable hybrid functional for a particular hybrid finite element $e$, i.e., element \#1 in Figure 4, can be expressed by [29].

$$
\Pi_{m e}=-\frac{1}{2} \int_{\Omega_{e}} k_{\mathrm{s}}\left(T_{\mathrm{s}, 1}^{2}+T_{\mathrm{s}, 2}^{2}\right) \mathrm{d} \Omega-\int_{\Gamma_{q e}} \bar{q} \widetilde{T}_{\mathrm{s}} \mathrm{d} \Gamma+\int_{\Gamma_{e}} q_{n}\left(\widetilde{T}_{\mathrm{s}}-T_{\mathrm{s}}\right) \mathrm{d} \Gamma-\frac{1}{2} \int_{\Gamma_{c e}} \widetilde{h}_{\infty}\left(\widetilde{T}_{\mathrm{s}}-\widetilde{T}_{\infty}\right)^{2} \mathrm{~d} \Gamma
$$

in which $\bar{q}$ represents the specified value of normal heat flux $q_{n}=q_{\mathrm{s}} \cdot n$ on the element heat flux boundary $\Gamma_{q e}, n=\left(n_{1}, n_{2}\right)$ is the unit normal vector to the element boundary, $T_{\mathrm{s}}$ and $\widetilde{T}_{\mathrm{s}}$ are respectively the temperature within the element and on the element boundary, $\Omega_{e}$ and $\Gamma_{e}$ respectively denote the element domain and element boundary, and $\Gamma_{c e}$ and $\Gamma_{q e}$ stand for the element convection boundary and heat flux boundary, respectively. It is noted that the four integral terms on the right side of Equation (22) represent the effects of thermal energy equilibrium, specified normal heat flux condition, inter-element continuity condition, and the convection condition, respectively. Besides, it is assumed that the coordinates $x_{1}$ and $x_{2}$ are set along the element plane. The temperature in the element is only a function of two space coordinates $x_{1}$ and $x_{2}$, when the prescribed temperature and heat flux are independent of the thickness direction of element. This means that the kinematics of heat flow along the thickness direction of the element can be ignored. Therefore, the two-dimensional hybrid finite element with thickness $t\left(x_{1}, x_{2}\right)$ can be used to model the computing domain. Here, the constant element thickness is considered in this work, for simplicity.

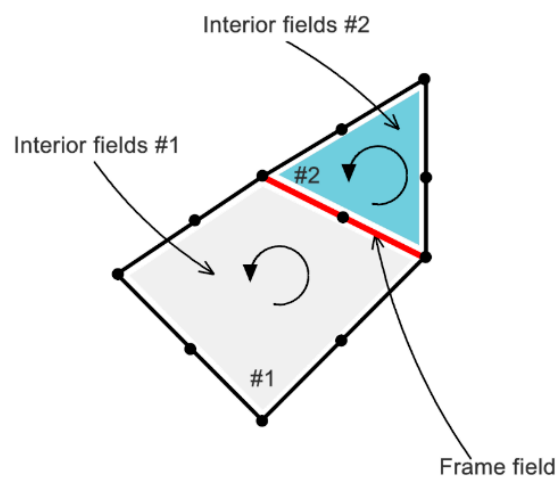

Figure 4. Schematic diagram of two-dimensional hybrid finite element.

In the application of variational functional (22), the temperature and its gradient fields at any point $x$ in the interior of the element $e$ are approximated by linear combination of fundamental solutions centered at series of source points $x_{j}^{\mathrm{s}}\left(j=1, \cdots, n_{\mathrm{s}}\right)$ locating on the pseudo boundary similar to the element boundary $\Gamma_{e}$, that is,

$$
\begin{gathered}
T_{\mathrm{s}}(x)=\sum_{j=1}^{n_{\mathrm{s}}} T^{*}\left(x, x_{j}^{s}\right) c_{e j}=N c_{e} \\
q_{\mathrm{s}}=-k_{\mathrm{s}} \nabla T_{\mathrm{s}}=-k_{\mathrm{s}} \nabla N c_{e}=T c_{e}
\end{gathered}
$$

where $c_{e}=\left\{c_{e j}\right\}^{T}\left(j=1, \cdots, n_{\mathrm{s}}\right)$ is the coefficient vector consisting of unknown source intensities at $n_{\mathrm{s}}$ source points locating outside the element domain. $N$ and $T$ are respectively coefficient matrices consisting of temperature fundamental solutions $T^{*}\left(x, x_{j}^{\mathrm{S}}\right)$ and heat flux fundamental solutions $q_{i}^{*}\left(x, x_{j}^{\mathrm{S}}\right)=-k_{\mathrm{s}} \partial T^{*}\left(x, x_{j}^{\mathrm{S}}\right) / \partial x_{i}(i=1,2)$ of problem under consideration, i.e.,

$$
\begin{aligned}
& N=\left[\begin{array}{llll}
T^{*}\left(x, x_{1}^{\mathrm{s}}\right) & T^{*}\left(x, x_{2}^{\mathrm{s}}\right) & \cdots & T^{*}\left(x, x_{n_{\mathrm{s}}}^{\mathrm{s}}\right)
\end{array}\right] \\
& T=\left[\begin{array}{llll}
q_{1}^{*}\left(x, x_{1}^{\mathrm{s}}\right) & q_{1}^{*}\left(x, x_{2}^{\mathrm{s}}\right) & \cdots & q_{1}^{*}\left(x, x_{n_{\mathrm{s}}}^{\mathrm{s}}\right) \\
q_{2}^{*}\left(x, x_{1}^{\mathrm{s}}\right) & q_{2}^{*}\left(x, x_{2}^{\mathrm{s}}\right) & \cdots & q_{2}^{*}\left(x, x_{n_{\mathrm{s}}}^{\mathrm{s}}\right)
\end{array}\right]
\end{aligned}
$$


For the two-dimensional isotropic and homogeneous substrate taken into consideration, the temperature fundamental solutions are given as [19].

$$
T^{*}\left(x, x_{j}^{\mathrm{s}}\right)=-\frac{1}{2 \pi k_{\mathrm{s}}} \ln r \quad \text { with } \quad r=\left\|x-x_{j}^{\mathrm{s}}\right\|
$$

Because the interior fields are independently defined for adjacent elements, i.e., element \#1 and \#2 in Figure 4, they should be weakly linked through the common interface. In order to enforce the conformity on the common interface of adjacent elements, the element frame temperature field $\widetilde{T}_{\mathrm{s}}$ can be defined in terms of the generalized nodal temperature vector $d_{e}=\left\{d_{i}\right\}^{T}\left(i=1, \cdots, n_{\mathrm{d}}\right)$ as

$$
\widetilde{T}_{\mathrm{S}}(x)=\sum_{i=1}^{n_{\mathrm{d}}} \widetilde{N}_{i}(x) d_{i}=\widetilde{N} d_{e}
$$

where $\widetilde{N}$ denotes the matrix consisting of the conventional one-dimensional shape functions widely used in the standard FEM [18] and BEM [19], $n_{\mathrm{d}}$ is the number of nodes of the element $e$.

The substitution of the interior and frame fields related to the element $e$ into the functional (22) gives

$$
\Pi_{m e}=-\frac{1}{2} c_{e}^{T} H_{e} c_{e}-d_{e}^{T} g_{e}+c_{e}^{T} G_{e} d_{e}-\frac{1}{2} d_{e}^{T} F_{e} d_{e}+d_{e}^{T} f_{e}-a_{e}
$$

where

$$
\begin{array}{ll}
H_{e}=\int_{\Gamma_{e}} Q^{T} N \mathrm{~d} \Gamma, & G_{e}=\int_{\Gamma_{e}} Q^{T} \widetilde{N} \mathrm{~d} \Gamma \\
F_{e}=\int_{\Gamma_{c e}} \widetilde{h}_{\infty} \widetilde{N}^{T} \widetilde{N} \mathrm{~d} \Gamma, & f_{e}=\int_{\Gamma_{c e}} \widetilde{h}_{\infty} \widetilde{T}_{\infty} \widetilde{N}^{T} \mathrm{~d} \Gamma \\
g_{e}=\int_{\Gamma_{e q}} \widetilde{N}^{T} \bar{q} \mathrm{~d} \Gamma, & a_{e}=\int_{\Gamma_{c e}} \frac{\widetilde{h}_{\infty} \widetilde{T}_{\infty}^{2}}{2} \mathrm{~d} \Gamma
\end{array}
$$

and $Q=A T$ with $A=\left\{n_{1} n_{2}\right\}$.

It is worth pointing out that during the procedure of deriving Equation (29) involving integrals along the element boundary only, the distinct feature that the interior fields exactly satisfy the governing equation is used to remove the domain integral in the functional (22). Moreover, the element boundary integrals in (29) mean that the polygonal element with more sides can be flexibly constructed for practical computation, not limited to triangle and quadrilateral elements in the conventional FEM.

Finally, the stationary conditions of $\Pi_{m e}$ with respect to the coefficient vector $c_{e}$ and the nodal temperature vector $d_{e}$,

$$
\begin{aligned}
\frac{\partial \Pi_{m e}}{\partial c_{e}^{T}} & =0 \\
\frac{\partial \Pi_{m e}}{\partial d_{e}^{T}} & =0
\end{aligned}
$$

yield the optional relationship between $c_{e}$ and $d_{e}$,

$$
c_{e}=H_{e}{ }^{-1} G_{e} d_{e}
$$

and the element stiffness equation,

$$
K_{e} d_{e}=g_{e}-f_{e}
$$

where

$$
K_{e}=G_{e}^{T} H_{e}^{-1} G_{e}-F_{e}
$$

It is clear from the above procedure that the element stiffness matrix is symmetric, and after assembling the global stiffness matrix keeps sparse and symmetric features, such as that in the conventional FEM. Additionally, the evaluation of the right-handed vector (33) is the same as that in the conventional FEM. Therefore, these features make the implementation of the present hybrid finite element conveniently incorporate into the existing FEM program. 


\section{Numerical Results}

In this section, five tests including the single-layered homogeneous coating with simple and complicated boundary conditions and the functionally graded coatings with exponential and linear graded forms are carried out for demonstrating the accuracy and applicability of the present method. Since the focus of the current work is on the development of generalized computational technique for analyzing various coating/substrate systems, the outmost material phase of the FGC layer or the single-layered homogeneous coating is arbitrarily assumed to have a thermal conductivity $6 \mathrm{~W} \mathrm{~m}^{-1} \mathrm{~K}^{-1}$. The thermal conductivity of the substrate material is assumed to have a value of $28 \mathrm{~W} \mathrm{~m}^{-1} \mathrm{~K}^{-1}$. These values can be adjusted to meet the practical coating/substrate system. Additionally, the convergence of the present hybrid finite element and the effect of the location of source points have been well investigated for thermal analysis (see literature [23,29-31] for reference), so they are not discussed in this study. Moreover, the 4 -sided hybrid finite element with 4 edges and 8 nodes is employed without exception in the following computation.

\subsection{Homogeneous Thin Coating with Simple Boundary Conditions}

Firstly, a simple test with a simple temperature boundary condition on the coating surface is performed to investigate the accuracy of the present method. In this test shown in Figure 5, it is assumed that both the left and right sides of the coating/substrate system are insulated, and the top and bottom sides are of specified temperature $f_{\mathrm{c}}$ and $f_{\mathrm{s}}$. From the basic heat transfer theory, the one-dimensional analytical solutions of temperature for the coating and substrate domains are given by

$$
\begin{array}{ll}
T_{\mathrm{c}}\left(x_{2}\right)=\frac{k_{\mathrm{s}}\left(f_{\mathrm{c}}-f_{\mathrm{s}}\right)}{k_{\mathrm{s}} h_{\mathrm{c}}+k_{\mathrm{c}} h_{\mathrm{s}}} x_{2}+\frac{f_{\mathrm{c}} h_{\mathrm{s}}\left(k_{\mathrm{c}}-k_{\mathrm{s}}\right)+f_{\mathrm{s}}\left(h_{\mathrm{c}}+h_{\mathrm{s}}\right) k_{\mathrm{s}}}{k_{\mathrm{s}} h_{\mathrm{c}}+k_{\mathrm{c}} h_{\mathrm{s}}}, & x_{2} \in \Omega_{\mathrm{c}} \\
T_{\mathrm{s}}\left(x_{2}\right)=\frac{k_{\mathrm{c}}\left(f_{\mathrm{c}}-f_{\mathrm{s}}\right)}{h_{\mathrm{s}}\left(k_{\mathrm{s}} \frac{h_{\mathrm{c}}}{h_{\mathrm{s}}}+k_{\mathrm{c}}\right)} x_{2}+f_{\mathrm{s}}, & x_{2} \in \Omega_{\mathrm{s}}
\end{array}
$$

from which it is seen that temperature distributions both in the coating and in the substrate are linear.

In the practical computation, it is assumed that the coating/substrate model is composed of a square substrate with $h_{\mathrm{s}}=1 \mathrm{~mm}$ and a thin homogeneous coating with thickness $h_{\mathrm{c}}$. The specified temperature boundary condition on the top and bottom surfaces of the model is assumed to be $f_{\mathrm{c}}=1173 \mathrm{~K}$ and $f_{\mathrm{s}}=298 \mathrm{~K}$, respectively. For the purpose of comparing the numerical and analytical results, the temperature distributions are evaluated in the substrate domain for different coating thickness values, which are controlled by the specific thickness ratio $h_{\mathrm{c}} / h_{\mathrm{s}}$ of the coating and substrate ranging from $10^{-6}$ to $10^{-1}$, and in each analysis, the hybrid finite element mesh with an element size of $0.1 \mathrm{~mm}$ is identically modelled. Since only the substrate domain is solved, the present approximated model is clearly more computationally efficient than the full-domain model consisting of the coating and substrate domains.

For different coating thickness values, the determined temperature values at three specific points respectively locating on the interface and within the substrate region, i.e., A $(0.4 \mathrm{~mm}, 1 \mathrm{~mm}), \mathrm{B}(0.2 \mathrm{~mm}$, $0.9 \mathrm{~mm})$ and C $(0.6 \mathrm{~mm}, 0.4 \mathrm{~mm})$, are recorded for analysis. Results in Table 1 indicate that there is perfect agreement between the numerical results and the analytical solutions, even for very small coating thicknesses. Thus, the present method shows a significant potential for analyzing thin coating problems. Moreover, it is seen that the temperature is nearly independent of the thickness ratio $h_{\mathrm{c}} / h_{\mathrm{s}}$ when its value is equal to or less than $10^{-4}$. It is reasonable that the extremely thin coating will make the temperature profile in the coating layer very close to the specified value $f_{\mathrm{c}}$.

However, it is worth noting that the test above is a one-dimensional linear case and it is not enough to assess the capability of the present method. Hence, more complex two-dimensional problems should be considered to check whether the present method can be used for them. 


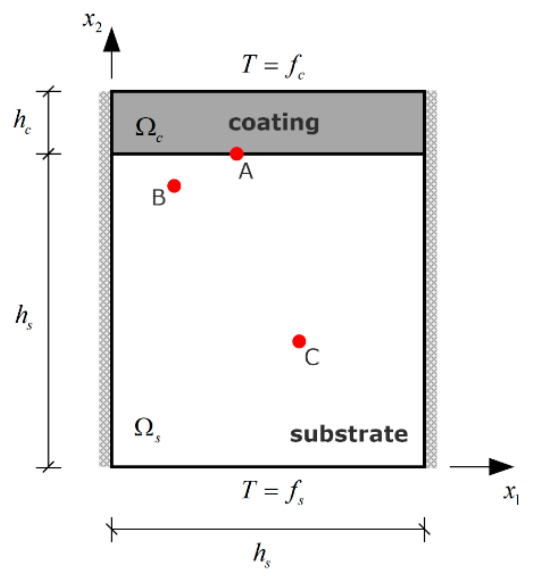

Figure 5. Configuration of the coating/substrate system in the first test.

Table 1. Temperature results at the three specific points for different thickness ratios in the first test.

\begin{tabular}{ccccccc}
\hline \multirow{2}{*}{$\boldsymbol{h}_{\mathrm{c}} / \boldsymbol{h}_{\mathbf{s}}$} & \multicolumn{2}{c}{ Point A } & \multicolumn{2}{c}{ Point B } & \multicolumn{2}{c}{ Point C } \\
\cline { 2 - 6 } & EXACT & Present & EXACT & Present & EXACT & Present \\
\hline $1 \times 10^{-6}$ & $1.1730 \times 10^{3}$ & $1.1730 \times 10^{3}$ & $1.0855 \times 10^{3}$ & $1.0855 \times 10^{3}$ & $6.4800 \times 10^{2}$ & $6.4800 \times 10^{2}$ \\
$1 \times 10^{-5}$ & $1.1730 \times 10^{3}$ & $1.1730 \times 10^{3}$ & $1.0855 \times 10^{3}$ & $1.0855 \times 10^{3}$ & $6.4798 \times 10^{2}$ & $6.4798 \times 10^{2}$ \\
$1 \times 10^{-4}$ & $1.1726 \times 10^{3}$ & $1.1726 \times 10^{3}$ & $1.0851 \times 10^{3}$ & $1.0851 \times 10^{3}$ & $6.4784 \times 10^{2}$ & $6.4784 \times 10^{2}$ \\
$1 \times 10^{-3}$ & $1.1689 \times 10^{3}$ & $1.1689 \times 10^{3}$ & $1.0818 \times 10^{3}$ & $1.0818 \times 10^{3}$ & $6.4637 \times 10^{2}$ & $6.4637 \times 10^{2}$ \\
$1 \times 10^{-2}$ & $1.1340 \times 10^{3}$ & $1.1340 \times 10^{3}$ & $1.0504 \times 10^{3}$ & $1.0504 \times 10^{3}$ & $6.3239 \times 10^{2}$ & $6.3239 \times 10^{2}$ \\
$1 \times 10^{-1}$ & $8.9459 \times 10^{2}$ & $8.9459 \times 10^{2}$ & $8.3493 \times 10^{2}$ & $8.3493 \times 10^{2}$ & $5.3664 \times 10^{2}$ & $5.3664 \times 10^{2}$ \\
\hline
\end{tabular}

\subsection{Homogeneous Thin Coating with Complicated Boundary Conditions}

In the second test, a complicated two-dimensional heat transfer is tested. The same geometrical domain and element mesh as those in the first test are taken here. Along the outer boundary of the coating/substrate system in Figure 5, the temperature boundary conditions are applied by the following exact solutions of temperature respectively for the coating and substrate regions

$$
\begin{array}{ll}
T_{\mathrm{c}}\left(x_{1}, x_{2}\right)=\eta x_{1}^{2}-\eta x_{2}^{2}+\frac{k_{\mathrm{s}}}{k_{\mathrm{c}}} x_{1} x_{2}+x_{1}+2 \eta x_{2}, & \left(x_{1}, x_{2}\right) \in \Omega_{\mathrm{c}} \\
T_{\mathrm{S}}\left(x_{1}, x_{2}\right)=\eta x_{1}^{2}-\eta x_{2}^{2}+x_{1} x_{2}+\frac{k_{\mathrm{s}}}{k_{\mathrm{c}}} x_{1}+2 \eta x_{2}, & \left(x_{1}, x_{2}\right) \in \Omega_{\mathrm{s}}
\end{array}
$$

where $\eta$ is any constant. It is obvious that Equation (36) is constructed by the polynomial including 2nd and 1st terms to satisfy the governing Equation (1) and the interfacial condition (5) so that the two-dimensional heat transfer in the coating/substrate system is ensured.

Firstly, we take $\eta=10$ in Equation (36) such that the analytical temperature distribution is independent of the thickness ratio $h_{\mathrm{c}} / h_{\mathrm{s}}$. Results at three different points the same as those in the first test are listed in Table 2, from which it is found that the accuracy of numerical results begins to slightly decrease as the thickness ratio drops below $10^{-2}$, and the relative error to the analytical solution at the interfacial point $A$ is the largest $(0.32 \%)$, compared to that at the interior points $B$ and C, i.e., $0.21 \%$ at the point $\mathrm{B}$ and $0.05 \%$ at the point $\mathrm{C}$. It is reasonable that the points $\mathrm{B}$ and $\mathrm{C}$ are far away from the interface on which the approximated transfer boundary conditions are applied. Besides, Figure 6 shows the variation of temperature along the interface and we observe that, although the temperature changes nonlinearly in terms of the coordinate $x_{1}$, the numerical results calculated by the present method are in good agreement with the exact solutions.

Subsequently, we take $\eta=1000 h_{\mathrm{c}} / h_{\mathrm{s}}$ so that the analytical temperature fields both in the coating and in the substitute severely depend on the thickness ratio $h_{\mathrm{c}} / h_{\mathrm{s}}$. Results in Table 3 indicate that the numerical results from the present method are still in good agreement with the exact solutions, although the values of temperature at the three specific points increase with the increase of the thickness 
ratio. Besides, the temperature variations along the interface for different thickness ratios are plotted in Figure 7 and the good agreement between the numerical results and the analytical solutions is observed again.

Table 2. Temperature results at the three specific points for different thickness ratios for $\eta=10$ in the second test.

\begin{tabular}{ccccccc}
\hline \multirow{2}{*}{$\boldsymbol{h}_{\mathrm{c}} / \boldsymbol{h}_{\mathbf{s}}$} & \multicolumn{2}{c}{ Point A } & \multicolumn{2}{c}{ Point B } & \multicolumn{2}{c}{ Point C } \\
\cline { 2 - 6 } & EXACT & Present & EXACT & Present & EXACT & Present \\
\hline $1 \times 10^{-6}$ & & 13.867 & & 11.413 & & 13.040 \\
$1 \times 10^{-5}$ & & 13.867 & & 11.413 & & 13.040 \\
$1 \times 10^{-4}$ & 13.867 & 13.867 & \multirow{2}{*}{11.413} & 11.413 & 13.040 & 13.040 \\
$1 \times 10^{-3}$ & & 13.867 & & 11.413 & & 13.040 \\
$1 \times 10^{-2}$ & & 13.866 & & 11.413 & & 13.040 \\
$1 \times 10^{-1}$ & & 13.822 & & 11.389 & & 13.033 \\
\hline
\end{tabular}

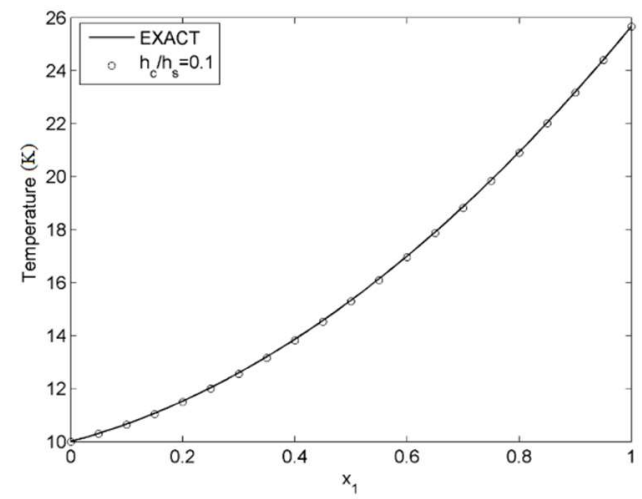

Figure 6. Variation of temperature along the interface for $\eta=10$ in the second test.

Table 3. Temperature results at the three specific points for different thickness ratios for $\eta=1000 h_{\mathrm{c}} / h_{\mathrm{S}}$ in the second test.

\begin{tabular}{ccccccc}
\hline \multirow{2}{*}{$\boldsymbol{h}_{\mathbf{c}} / \boldsymbol{h}_{\mathbf{s}}$} & \multicolumn{2}{c}{ Point A } & \multicolumn{2}{c}{ Point B } & \multicolumn{2}{c}{ Point C } \\
\cline { 2 - 7 } & EXACT & Present & EXACT & Present & EXACT & Present \\
\hline $1 \times 10^{-6}$ & 2.2678 & 2.2678 & 1.1144 & 1.1144 & 3.0410 & 3.0410 \\
$1 \times 10^{-5}$ & 2.2783 & 2.2783 & 1.1236 & 1.1236 & 3.0500 & 3.0500 \\
$1 \times 10^{-4}$ & 2.3827 & 2.3827 & 1.2163 & 1.2163 & 3.1400 & 3.1400 \\
$1 \times 10^{-3}$ & 3.4267 & 3.4267 & 2.1433 & 2.1433 & 4.0400 & 4.0400 \\
$1 \times 10^{-2}$ & 13.867 & 13.866 & 11.413 & 11.413 & 13.040 & 13.040 \\
$1 \times 10^{-1}$ & 118.27 & 117.82 & 104.11 & 103.87 & 103.04 & 102.97 \\
\hline
\end{tabular}

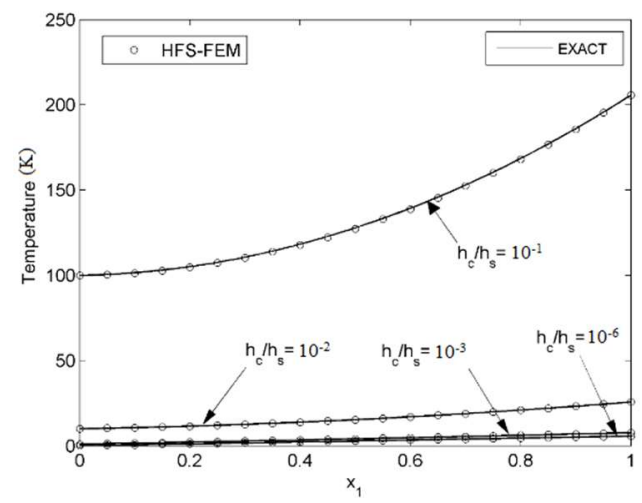

Figure 7. Variation of temperature along the interface for $\eta=1000 h_{\mathrm{c}} / h_{\mathrm{S}}$ in the second test. 


\subsection{Partially Heated Homogeneous Thin Coating}

A substrate with a partially heated homogeneous thin coating is considered in the third test. The boundary conditions specified in Figure 8 a are complicated enough to ensure two-dimensional heat transfer. The side length $h_{s}$ of the square substrate domain is $7 \mathrm{~mm}$, and the local length $l_{0}$ of the specific extremely high temperature constraint is $1.75 \mathrm{~mm}$. The related hybrid finite element mesh in the substrate domain is generated with 700 elements, as shown in Figure 8b.

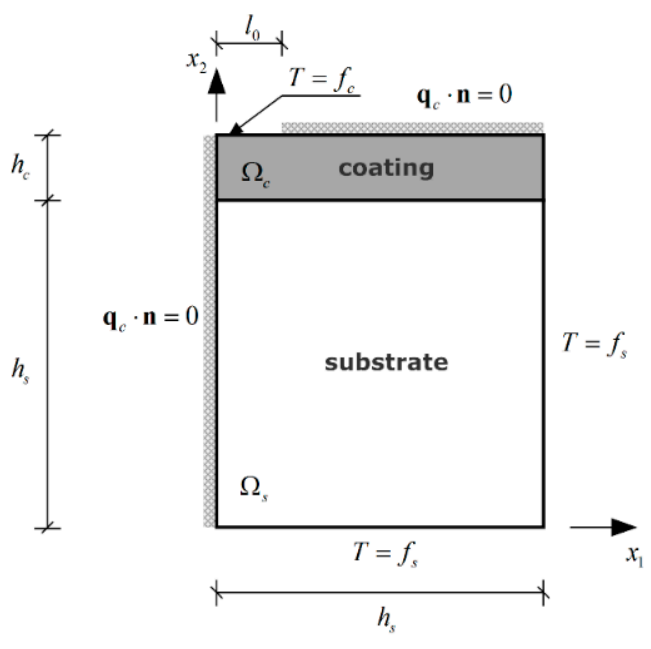

(a)

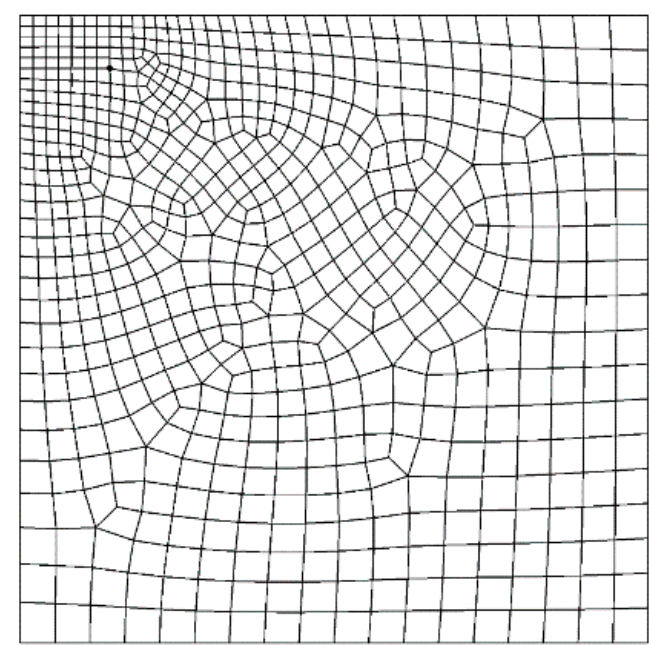

(b)

Figure 8. (a) Configuration of the coating/substrate system in the third test, (b) the in-plane hybrid finite element mesh in the substrate domain.

Because there is no analytical solution for this test, the numerical results from the present method are compared to the available BEM solutions [13]. To do so, the determined temperature values at the point $(1 \mathrm{~mm}, 6.41 \mathrm{~mm})$ within the substrate region are recorded in Table 4 for comparison under different coating thicknesses between $1 \mu \mathrm{m}$ to $1 \mathrm{~mm}$. From Table 4 , it is shown that the deviation between numerical results from the single-domain BEM and the present method can be ignored. Thus, the present method can be used for engineering analysis of thin coating. Besides, it is seen from Table 4 that, when the coating thickness is less than $10 \mu \mathrm{m}$, the temperature at the specific point just changes slightly. This can be attributed to the extremely small thickness of the coating layer.

Table 4. Results at the specific point for different coating thickness in the third test.

\begin{tabular}{ccc}
\hline $\boldsymbol{h}_{\mathbf{c}}(\boldsymbol{\mu \mathrm { m } )}$ & BEM [13] & Present \\
\hline 0.01 & - & 1016.1 \\
0.1 & - & 1.015 .9 \\
0.5 & - & 1015.2 \\
1 & 1016.5 & 1014.3 \\
2 & 1014.8 & 1012.6 \\
3 & 1013.2 & 1010.9 \\
5 & 1010 & 1007.8 \\
10 & 1002.4 & 1000.5 \\
50 & 951.2 & 950.1 \\
500 & 666.5 & 666.1 \\
1000 & 547 & 546.7 \\
\hline
\end{tabular}

Note: - means unavailable result. 


\subsection{Linear-Form Functionally Graded Coating}

Different to the conventional homogeneous coating studied above, the FGC offers the possibility of significantly improving interface reliability caused by material mismatch [17], and thus is considered in this and the next tests. Identical geometry and boundary conditions to that depicted in Figure 5 are employed. It is assumed that the thermal conductivity of the FGC varies along the thickness of coating in the following linear form:

$$
k_{\mathrm{c}}\left(x_{2}\right)=a+b x_{2}, \quad h_{\mathrm{s}} \leq x_{2} \leq h_{\mathrm{c}}+h_{\mathrm{s}}
$$

in which the coefficients $a$ and $b$ can be determined from the continuous change of thermal conductivity from the outmost material phase $k_{\mathrm{c} 0}=6 \mathrm{~W} \mathrm{~m}^{-1} \mathrm{~K}^{-1}$ to the inner substrate material phase $k_{\mathrm{S}}=28 \mathrm{~W} \mathrm{~m}^{-1} \mathrm{~K}^{-1}$, i.e., when $x_{2}=h_{\mathrm{c}}+h_{\mathrm{s}}, k_{\mathrm{c}}=k_{\mathrm{c} 0}$, and when $x_{2}=h_{\mathrm{s}}, k_{\mathrm{c}}=k_{\mathrm{s}}$, from which we have

$$
a=\left(1+\frac{h_{\mathrm{s}}}{h_{\mathrm{c}}}\right) k_{\mathrm{s}}-\frac{h_{\mathrm{s}}}{h_{\mathrm{c}}} k_{\mathrm{c} 0}, \quad b=\frac{k_{\mathrm{c} 0}-k_{\mathrm{s}}}{h_{\mathrm{c}}}
$$

From Equation (38), it is seen that the value of $a$ is linearly dependent of the thickness ratio $h_{\mathrm{s}} / h_{\mathrm{c}}$, but $b$ depends on the coating thickness only. For example, for $h_{\mathrm{c}}=0.001 \mathrm{~mm}$, we have $a=22,028$ and $b=-22,000$.

From the basic heat transfer theory, the one-dimensional analytical solution of temperature in the coating/substrate system can be derived by substituting Equation (37) into Equation (4),

$$
\begin{array}{rlrl}
T_{\mathrm{c}}= & \frac{k_{\mathrm{s}}\left(f_{\mathrm{c}}-f_{\mathrm{s}}\right)}{k_{\mathrm{s}}\left\{\ln \left[a+\left(h_{\mathrm{c}}+h_{\mathrm{s}}\right) b\right]-\ln \left(a+h_{\mathrm{s}} b\right)\right\}+b h_{\mathrm{s}}} \ln \left(a+b x_{2}\right)+ & \\
& \frac{k_{\mathrm{s}} f_{\mathrm{s}} \ln \left[a+\left(h_{\mathrm{c}}+h_{\mathrm{s}}\right) b\right]-f_{\mathrm{c}} k_{\mathrm{s}} \ln \left(a+h_{\mathrm{s}} b\right)+f_{\mathrm{c}} h_{\mathrm{s}} b}{k_{\mathrm{s}}\left\{\ln \left[a+\left(h_{\mathrm{c}}+h_{\mathrm{s}}\right) b\right]-\ln \left(a+h_{\mathrm{s}} b\right)\right\}+b h_{\mathrm{s}}}, & & h_{\mathrm{s}} \leq x_{2} \leq h_{\mathrm{s}}+h_{\mathrm{c}} \\
T_{\mathrm{s}}= & \frac{b\left(f_{\mathrm{c}}-f_{\mathrm{s}}\right)}{k_{\mathrm{s}}\left\{\ln \left[a+\left(h_{\mathrm{c}}+h_{\mathrm{s}}\right) b\right]-\ln \left(a+h_{\mathrm{s}} b\right)\right\}+b h_{\mathrm{s}}} x_{2}+f_{\mathrm{s}}, & 0 \leq x_{2} \leq h_{\mathrm{s}}
\end{array}
$$

To demonstrate the capability of the present method for analyzing this inhomogeneous coating, the coating is uniformly divided into 2, 6 and 10 thin layers, respectively, and each layer is assumed to be homogeneous. The related thermal conductivity of each layer is evaluated by the coordinate of centroid of the layer. Table 5 demonstrates the temperature results at the interfacial point $(0.5 \mathrm{~mm}$, $1 \mathrm{~mm}$ ) for different coating thicknesses. It is clearly seen that the numerical accuracy of the present method becomes better as the number of layers increases for each value of the coating thickness. Besides, for $M=2$, the results deteriorate quickly as the coating thickness is less than $0.1 \mathrm{~mm}$; however, more layers, i.e., $M=6$ and $M=10$, can produce very consistent results with the exact solutions even though the thickness is larger than $0.1 \mathrm{~mm}$. Finally, the temperature variations along the middle line $x_{1}=0.5 \mathrm{~mm}$ for different coating thicknesses are plotted in Figure 9 when the 10-layered coating model is employed. It is found that the temperature profile in the substrate tends to be unchanged as the thickness of the coating becomes small.

Table 5. Temperature at the interfacial point $(0.5 \mathrm{~mm}, 1 \mathrm{~mm})$ for different coating thicknesses for linear gradation.

\begin{tabular}{ccccc}
\hline $\boldsymbol{h}_{\mathrm{c}}(\mathbf{m m})$ & $\boldsymbol{M}=\mathbf{2}$ & $\boldsymbol{M}=\mathbf{6}$ & $\boldsymbol{M}=\mathbf{1 0}$ & EXACT \\
\hline 0.001 & 1171.4 & 1171.3 & 1171.3 & 1171.3 \\
0.01 & 1157.2 & 1156.3 & 1156.2 & 1156.2 \\
0.1 & 1037.0 & 1030.7 & 1030.0 & 1029.6 \\
0.2 & 937.65 & 928.15 & 927.14 & 926.54 \\
0.3 & 861.83 & 850.81 & 849.64 & 848.95 \\
\hline
\end{tabular}




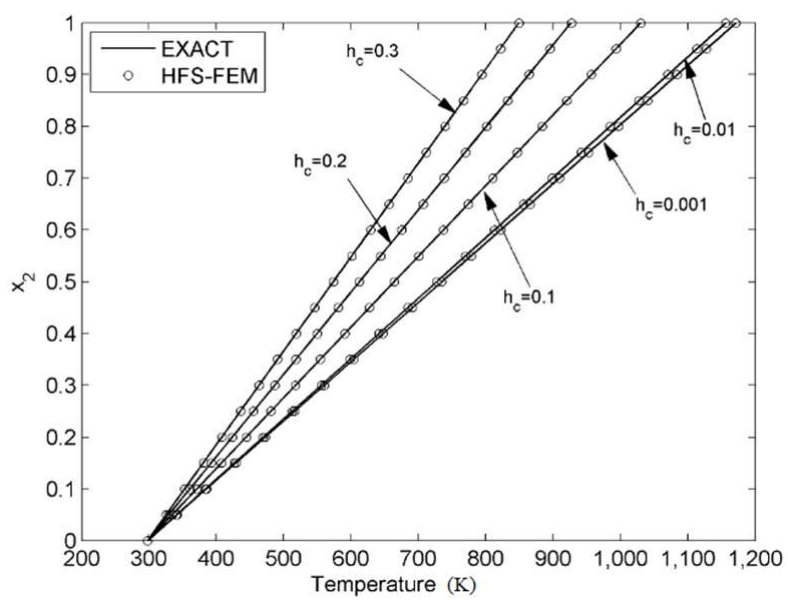

Figure 9. Temperature variation along the middle line $x_{1}=0.5 \mathrm{~mm}$ for different coating thicknesses for linear gradation.

\subsection{Exponential-Form Functionally Graded Coating}

In the last test, the same model as that in the fourth test is considered and the exponent form,

$$
k_{\mathrm{c}}\left(x_{2}\right)=a e^{b x_{2}}, \quad h_{\mathrm{s}} \leq x_{2} \leq h_{\mathrm{c}}+h_{\mathrm{s}}
$$

is utilized to represent the continuous change of the thermal conductivity of the coating material. The coefficients $a$ and $b$ are similarly determined from the continuous change of thermal conductivity from the outmost material phase $k_{\mathrm{c} 0}=6 \mathrm{~W} \mathrm{~m}^{-1} \mathrm{~K}^{-1}$ to the inner substrate material phase $k_{\mathrm{s}}=28 \mathrm{~W} \mathrm{~m}^{-1} \mathrm{~K}^{-1}$, i.e., when $x_{2}=h_{\mathrm{c}}+h_{\mathrm{s}}, k_{\mathrm{c}}=k_{\mathrm{c} 0}$, and when $x_{2}=h_{\mathrm{s}}, k_{\mathrm{c}}=k_{\mathrm{s}}$, from which we have

$$
a=k_{\mathrm{c} 0} e^{\left[-\left(\frac{h_{\mathrm{s}}}{h_{\mathrm{c}}}+1\right) \ln \frac{k_{\mathrm{c} 0}}{k_{\mathrm{s}}}\right]}, \quad b=\frac{1}{h_{\mathrm{c}}} \ln \frac{k_{\mathrm{c} 0}}{k_{\mathrm{s}}}
$$

From Equation (41), it is seen that the value of $a$ is seriously dependent on the thickness ratio $h_{\mathrm{c}} / h_{\mathrm{s}}$, while the coefficient $b$ depends on the coating thickness only. Too small $h_{\mathrm{c}} / h_{\mathrm{s}}$ will cause extremely large $a$, which is not suitable for practical use. For example, for the case of $h_{\mathrm{c}}=0.1 \mathrm{~mm}, h_{\mathrm{s}}=1 \mathrm{~mm}$, we have $a=1.3716 \times 10^{8}, \mathrm{~b}=-15.4045$.

From the basic heat transfer theory, the one-dimensional analytical solution of temperature in the coating and substrate domains can be derived by substituting Equation (40) into Equation (4)

$$
\begin{aligned}
& T_{\mathrm{c}}=-\frac{1}{e^{a x_{2}}} \frac{e^{b\left(2 h_{\mathrm{s}}+h_{\mathrm{c}}\right)} k_{\mathrm{s}}\left(f_{\mathrm{c}}-f_{\mathrm{s}}\right)}{a b e^{b\left(2 h_{\mathrm{s}}+h_{\mathrm{c}}\right)} h_{\mathrm{s}}+k_{\mathrm{s}}\left(e^{b\left(h_{\mathrm{s}}+h_{\mathrm{c}}\right)}-e^{b h_{\mathrm{s}}}\right)}+\frac{a b e^{b\left(2 h_{\mathrm{s}}+h_{\mathrm{c}}\right)} f_{\mathrm{c}} h_{\mathrm{s}}+f_{\mathrm{c}} k_{\mathrm{s}} e^{b\left(h_{\mathrm{s}}+h_{\mathrm{c}}\right)}-f_{\mathrm{s}} k_{\mathrm{s}} e^{b h_{\mathrm{s}}}}{a b e^{b\left(2 h_{\mathrm{s}}+h_{\mathrm{c}}\right)} h_{\mathrm{s}}+k_{\mathrm{s}}\left(e^{b\left(h_{\mathrm{s}}+h_{\mathrm{c}}\right)}-e^{b h_{\mathrm{s}}}\right)}, \\
& T_{\mathrm{S}}=\frac{a b e^{b\left(2 h_{\mathrm{s}}+h_{\mathrm{c}}\right)}\left(f_{\mathrm{c}}-f_{\mathrm{s}}\right)}{a b e^{b\left(2 h_{\mathrm{s}}+h_{\mathrm{c}}\right)} h_{\mathrm{s}}+k_{\mathrm{s}}\left(e^{b\left(h_{\mathrm{s}}+h_{\mathrm{c}}\right)}-e^{b h_{\mathrm{s}}}\right)} x_{2}+f_{\mathrm{s}} \\
& \begin{aligned}
h_{\mathrm{s}} & \leq x_{2} \leq h_{\mathrm{s}}+h_{\mathrm{c}} \\
& 0 \leq x_{2} \leq h_{\mathrm{s}}
\end{aligned}
\end{aligned}
$$

To demonstrate the performance of the present method for exponentially graded coating, the coating is also divided into 2, 6, 10 thin layers and each layer is assumed to be homogeneous. When the coating thickness changes from 0.1 to $0.3 \mathrm{~mm}$, the temperature results calculated from the present method both in Table 6 and in Figure 10 show good agreement with the analytical solutions. Moreover, the convergent tendency of temperature can be observed from Table 6 when the number of layers increases, as expected. Additionally, in contrast to the results in Table 5, it is seen that the exponentially graded distribution can produce a smaller temperature at the interface than the linear distribution under the same coating thickness. This is because the exponential variation of thermal conductivity can yield a smaller value than the linear form. 
Table 6. Temperature at the interfacial point $(0.5 \mathrm{~mm}, 1 \mathrm{~mm})$ for different coating thicknesses for exponential gradation.

\begin{tabular}{ccccc}
\hline$h_{\mathrm{c}}(\mathbf{m m})$ & $\boldsymbol{M}=\mathbf{2}$ & $\boldsymbol{M}=\mathbf{6}$ & $\boldsymbol{M}=\mathbf{1 0}$ & EXACT \\
\hline 0.1 & 1008.1 & 1005.1 & 1004.9 & 1004.8 \\
0.2 & 895.47 & 891.32 & 890.99 & 890.80 \\
0.3 & 813.70 & 809.06 & 808.68 & 808.48 \\
\hline
\end{tabular}

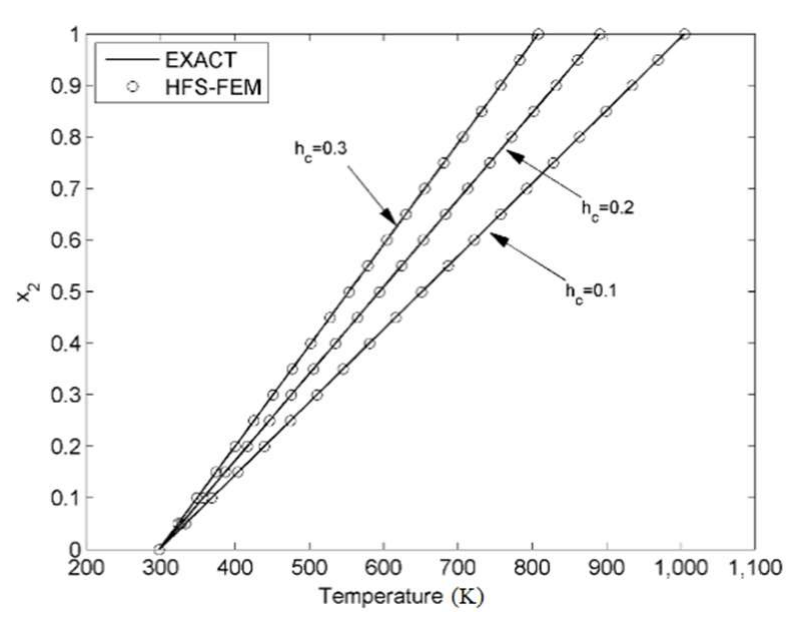

Figure 10. Temperature variation along the middle line $x_{1}=0.5 \mathrm{~mm}$ for different coating thicknesses (10 layers) for exponential gradation.

\section{Conclusions}

In this work, the general functionally graded coating/substrate system is solved by integrating the approximated transfer algorithm and the HFS-FEM for predicting the temperature distribution in the substrate and numerical results, demonstrating that the proposed method can be applied to various coatings with high computational efficiency and accuracy. In contrast to the previous works in the literature, the proposed method can be summarized as follows:

- The approximated recurrence formula based on the finite difference technique is derived for approximately transferring the general thermal boundary condition applied on the coating surface to the coating/substrate interface.

- The established transfer approach is highly suitable for treating thin multi-layered coating or functionally graded coating.

- With the present transfer approach, only the substrate needs to be solved, instead of the whole coating/substrate system.

- With the fundamental solutions of the problem as kernel functions, the HFS-FEM involving the evaluation of element boundary integrals only is implemented in the substrate region to achieve high accuracy and feasible element construction.

Author Contributions: Conceptualization, H.W. and Q.H.; Methodology, H.W. and Q.H.; Validation, H.W. and Q.H.; Formal Analysis, H.W. and Q.H.; Writing-Original Draft Preparation, H.W. and Q.H.; Writing-Review \& Editing, H.W. and Q.H.

Funding: The research was funded by the National Natural Science Foundation of China (No. 11472099), the fund of the State Key Laboratory of Structural Analysis for Industrial Equipment in the Dalian University of Technology (No. GZ1610) and the Program for Innovative Research Team of Science \& Technology of Henan Province of China (No. 19IRTSTHN020).

Conflicts of Interest: The authors declare no conflict of interest. 


\section{References}

1. Liu, Z.; Meyers, M.A.; Zhang, Z.; Ritchie, R.O. Functional gradients and heterogeneities in biological materials: Design principles, functions, and bioinspired applications. Prog. Mater. Sci. 2017, 88, 467-498. [CrossRef]

2. Mehrali, M.; Shirazi, F.S.; Mehrali, M.; Metselaar, H.S.C.; Kadri, N.A.B.; Osman, N.A.A. Dental implants from functionally graded materials. J. Biomed. Mater. Res. A 2013, 101, 3046-3057. [CrossRef]

3. Habibi, M.K.; Samaei, A.T.; Gheshlaghi, B.; Lu, J.; Lu, Y. Asymmetric flexural behavior from bamboo's functionally graded hierarchical structure: Underlying mechanisms. Acta Biomater. 2015, 16, $178-186$. [CrossRef] [PubMed]

4. Askarinejad, S.; Rahbar, N. Toughening mechanisms in bioinspired multilayered materials. J. R. Soc. Interface 2015, 12, 20140855. [CrossRef]

5. Pompe, W.; Worch, H.; Epple, M.; Friess, W.; Gelinsky, M.; Greil, P.; Hempel, U.; Scharnweber, D.; Schulte, K. Functionally graded materials for biomedical applications. Mater. Sci. Eng. A 2003, 362, 40-60. [CrossRef]

6. Roop Kumar, R.; Wang, M. Functionally graded bioactive coatings of hydroxyapatite/titanium oxide composite system. Mater. Lett. 2002, 55, 133-137. [CrossRef]

7. Padture, N.P.; Gell, M.; Jordan, E.H. Thermal barrier coatings for gas-turbine engine applications. Science 2002, 296, 280-284. [CrossRef]

8. Cinefra, M.; Valvano, S.; Carrera, E. Heat conduction and thermal stress analysis of laminated composites by a variable kinematic MITC9 shell element. Curved Lay. Struct. 2015, 2, 301-320. [CrossRef]

9. Brischetto, S.; Leetsch, R.; Carrera, E.; Wallmersperger, T.; Kröplin, B. Thermo-mechanical bending of functionally graded plates. J. Therm. Stress. 2008, 31, 286-308. [CrossRef]

10. Carrera, E.; Valvano, S. A variable kinematic shell formulation applied to thermal stress of laminated structures. J. Therm. Stress. 2017, 40, 803-827. [CrossRef]

11. Buyukkaya, E.; Cerit, M. Thermal analysis of a ceramic coating diesel engine piston using 3-D finite element method. Surf. Coat. Technol. 2007, 202, 398-402. [CrossRef]

12. Grzesik, W.; Bartoszuk, M.; Nieslony, P. Finite element modelling of temperature distribution in the cutting zone in turning processes with differently coated tools. J. Mater. Process. Technol. 2005, 164, 1204-1211. [CrossRef]

13. Du, F.; Lovell, M.R.; Wu, T.W. Boundary element method analysis of temperature fields in coated cutting tools. Int. J. Solids Struct. 2001, 38, 4557-4570. [CrossRef]

14. Zhang, Y.; Gu, Y.; Chen, J.T. Boundary element analysis of the thermal behaviour in thin-coated cutting tools. Eng. Anal. Bound. Elem. 2010, 34, 775-784. [CrossRef]

15. Gu, Y.; Chen, W.; Zhang, C. Stress analysis for thin multilayered coating systems using a sinh transformed boundary element method. Int. J. Solids Struct. 2013, 50, 3460-3471. [CrossRef]

16. Buyukkaya, E. Thermal analysis of functionally graded coating AlSi alloy and steel pistons. Surf. Coat. Technol. 2008, 202, 3856-3865. [CrossRef]

17. Lee, W.Y.; Stinton, D.P.; Berndt, C.C.; Erdogan, F.; Lee, Y.D.; Mutasim, Z. Concept of functionally graded materials for advanced thermal barrier coating applications. J. Am. Ceram. Soc. 1996, 79, 3003-3012. [CrossRef]

18. Zienkiewicz, O.C.; Taylor, R.L.; Zienkiewicz, O.C.; Taylor, R.L. The Finite Element Method; McGraw-Hill: London, UK, 1977.

19. Brebbia, C.A.; Telles, J.C.F.; Wrobel, L. Boundary Element Techniques: Theory and Applications in Engineering; Springer: Berlin, Gremnay, 1984.

20. Qin, Q.H. Nonlinear analysis of reissner plates on an elastic foundation by the BEM. Int. J. Solids Struct. 1993, 30, 3101-3111. [CrossRef]

21. Qin, Q.H.; Mai, Y.W. BEM for crack-hole problems in thermopiezoelectric materials. Eng. Fract. Mech. 2002, 69, 577-588. [CrossRef]

22. Wang, H.; Qin, Q.H. Fundamental-solution-based hybrid FEM for plane elasticity with special elements. Comput. Mech. 2011, 48, 515-528. [CrossRef]

23. Wang, H.; Qin, Q.H. Hybrid FEM with fundamental solutions as trial functions for heat conduction simulation. Acta Mech. Solida Sin. 2009, 22, 487-498. [CrossRef]

24. Mitchell, A.R.; Griffiths, D.F. The Finite Difference Method in Partial Differential Equations; John Wiley: New York, NY, USA, 1980. 
25. Qin, Q.H. Solving anti-plane problems of piezoelectric materials by the Trefftz finite element approach. Comput. Mech. 2003, 31, 461-468. [CrossRef]

26. Qin, Q.H.; Wang, H. MATLAB and C Programming for Trefftz Finite Element Methods; CRC Press: Boca Raton, FL, USA, 2009.

27. Wang, H.; Qin, Q.H. A new special element for stress concentration analysis of a plate with elliptical holes. Acta Mech. 2012, 223, 1323-1340. [CrossRef]

28. Wang, H.; Qin, Q.H. Fundamental-solution-based finite element model for plane orthotropic elastic bodies. Eur. J. Mech.-A/Solids 2010, 29, 801-809. [CrossRef]

29. Wang, H.; Qin, Q.H. FE approach with Green's function as internal trial function for simulating bioheat transfer in the human eye. Arch. Mech. 2010, 62, 493-510.

30. Wang, H.; Zhao, X.; Wang, J. Interaction analysis of multiple coated fibers in cement composites by special n-sided interphase/fiber elements. Compos. Sci. Technol. 2015, 118, 117-126. [CrossRef]

31. Wang, H.; Qin, Q.H.; Xiao, Y. Special n-sided Voronoi fiber/matrix elements for clustering thermal effect in natural-hemp-fiber-filled cement composites. Int. J. Heat Mass Transf. 2016, 92, 228-235. [CrossRef]

(C) 2019 by the authors. Licensee MDPI, Basel, Switzerland. This article is an open access article distributed under the terms and conditions of the Creative Commons Attribution (CC BY) license (http://creativecommons.org/licenses/by/4.0/). 